\title{
A Generic Model for Predicting the Permeability of Fiber Mats Chensong Dong ${ }^{1, a}$ and Tuong Yung Tang ${ }^{1, b}$ \\ ${ }^{1}$ Department of Mechanical Engineering, Curtin University of Technology, GPO Box U1987, Perth, WA 6845, Australia \\ ac.dong@curtin.edu.au,
}

Keywords: Fiber; permeability; model

\begin{abstract}
A new empirical model for predicting the permeability of fiber mats is presented in this paper. Permeability data were collected from the NIST reinforcement permeability database and categorized according to the material architecture. It is seen from the data that for each category, permeability is proportional to fiber volume fraction. In order to describe the behavior of permeability vs. fiber volume fraction, the location, scale and shape parameters were introduced for each material category. The model was validated against the experimental data and good agreement was found.
\end{abstract}

\section{Introduction}

Liquid composite molding (LCM), which includes resin transfer molding (RTM), vacuum assisted RTM (VARTM), reaction injection molding (RIM) and resin infusion, is considered one of the most viable and environmentally benign solutions for fabricating high quality and affordable composite materials. Extensive research has been carried out on the development of process models and computer simulation tools for predicting LCM process behaviors [1]. Using these models and tools, resin flow behavior can be predicted. Computer simulation tools provide an important mechanism for LCM process design optimization.

Permeability is the property of fibers that indicates the degree of ease with which fluids travel through the pore space and impregnates the fibers. It is necessary to characterize the permeability of fiber mats to simulate the resin flow phenomenon during LCM processes. Without an accurate estimation of the fiber preform permeability profile, simulation models are unable provide useful information for process design.

During the past decade, many research studies have reported on permeability characterization. A progress review is presented by Abrate [2]. The permeability of various fiber structures has been investigated. These research studies have provided a fundamental understanding and some experimental results of permeability through analytical and experimental investigations.

Permeability measurement is usually performed based on either a channel flow or a radial flow [2]. For both cases, methods based on either saturated or unsaturated flow measurements have been proposed.

Correct estimation of the permeability is crucial for the liquid composite molding process simulation. NIST has developed a permeability database for standard reference fabrics [3]. Despite this, empirical models for permeability prediction vary and there lacks a generic model, which is useful for predicting the permeability of any type of fiber mats. In this paper, the data from the NIST database were fit to several empirical models. Based on the comparison of these empirical models, a new empirical model was developed. This model was validated against the experimental data. It is shown that this new model provides better overall accuracy of permeability prediction for all types of fiber mats.

\section{Data Collection}

In this study, permeability data were collected from the NIST reinforcement permeability database. 90 data from the unidirectional saturated measurement, 16 data from the radial unsaturated measurement, and 9 data from the through-thickness measurement were collected. For each data, 
the permeability and parameters including flow direction relative to fiber geometry, fiber volume fraction, test fluid viscosity, material category and material construction were recorded.

Because the through-thickness permeability of a fiber preform is usually much lower than the inplane permeability, the data from the unidirectional, radial and through-thickness measurements were analyzed separately. For each measurement, the data were organized into groups according to the material category and construction, as shown in Table 1. It is seen a general trend is that permeability is inversely proportional to fiber volume fraction.

Table 1: Groups of permeability data

\begin{tabular}{clll}
\hline Group & Flow & Material category & Material construction \\
\hline 1 & Unidirectional & Woven & 8-harness satin \\
2 & Unidirectional & Woven & 5-harness satin \\
3 & Unidirectional & Woven & Crow foot \\
4 & Unidirectional & 3D woven & Layer interlock \\
5 & Unidirectional & Random & 3 sub-layers \\
6 & Unidirectional & Non-woven stitched & Double bias $\pm 45^{\circ}$ \\
7 & Unidirectional & Unidirectional & Weft unidirectional or woven warp unidirectional \\
8 & Radial & 3D woven & Layer interlock \\
9 & Radial & Random & 3 sub-layers \\
10 & Radial & Unidirectional & Weft unidirectional or woven warp unidirectional \\
11 & Through-thickness & Random & 3 sub-layers \\
12 & Through-thickness & Unidirectional & Weft unidirectional or woven warp unidirectional \\
\hline
\end{tabular}

\section{Model Development}

A comparison of the empirical models in the literature shows that these models yield similar permeability values. However, when extrapolated, some models yield negative permeability values at low fiber volume fractions while others over-estimate the permeability values. In addition, some of the models are very sophisticated so that they are difficult to be used as a generic model.

If permeability is plotted vs. porosity, as shown in Fig. 1 for the 8-harness satin fiber mats, the pattern suggests the following relationship can be used to develop a new empirical model.

$$
K=a\left(\phi-\phi_{0}\right)^{b}
$$

where $K$ is permeability; $\phi$ is porosity; $\phi_{0}$ is the cut-off porosity; and $a$ and $b$ are constants.

Alternatively, Eqn. (1) can be written as

$$
K=a\left(V_{f 0}-V_{f}\right)^{b}
$$

where $V_{f 0}$ is the cut-off fiber volume fraction, i.e. the maximum fiber volume fraction that can be achieved for this type of fiber mats. This can be regarded as the location parameter, and $a$ and $b$ are the scale and shape parameters, respectively.

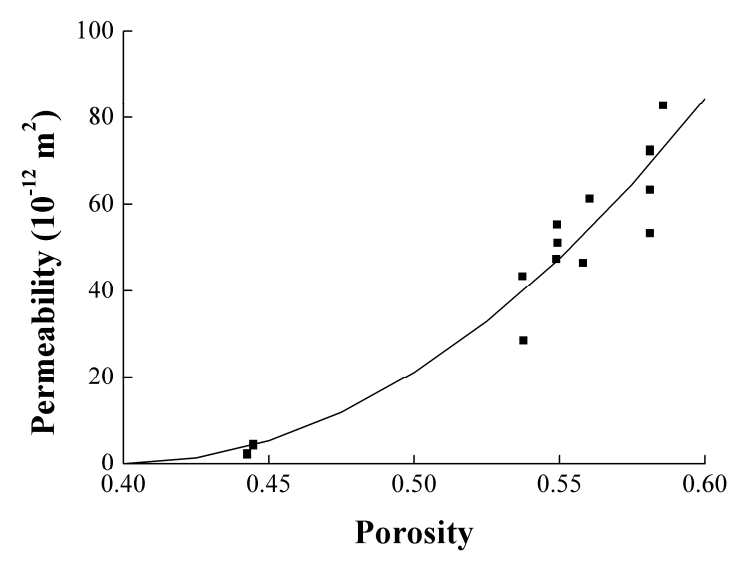

Fig. 1: Permeability vs. porosity for 8-harness satin fiber mats 
The data collected from the database were fitted to the empirical model as shown in Eqn. (2). Because of the number of data, only the data from the unidirectional and through-thickness measurements were used for model development. The model parameters are shown in Table 2.

Table 2: Model parameters

\begin{tabular}{|c|c|c|c|c|c|}
\hline Group & Flow & Material category & $a$ & $\overline{V_{f 0}}$ & $b$ \\
\hline 1 & Unidirectional & Woven: 8-harness satin & 2110 & 0.6 & 2 \\
\hline 2 & Unidirectional & Woven: 5-harness satin & 3585 & 0.6 & 1.73 \\
\hline 3 & Unidirectional & Woven: Crow foot & 1245 & 0.7 & 1.58 \\
\hline 4 & Unidirectional & $\begin{array}{l}\text { 3D woven: Layer } \\
\text { interlock }\end{array}$ & 7180 & 0.6 & 1.79 \\
\hline 5 & Unidirectional & Random: 3 sub-layers & 548626 & 0.4 & 3.22 \\
\hline 6 & Unidirectional & $\begin{array}{l}\text { Non-woven stitched: } \\
\text { Double bias } \pm 45^{\circ}\end{array}$ & 4300 & 0.6 & 1.83 \\
\hline 7 & Unidirectional & $\begin{array}{l}\text { Unidirectional: Weft } \\
\text { unidirectional or woven } \\
\text { warp unidirectional }\end{array}$ & 960 & 0.7 & 1 \\
\hline 11 & Through-thickness & Random: 3 sub-layers & 7569 & 0.4 & 2.2 \\
\hline 12 & Through-thickness & $\begin{array}{l}\text { unidirectional or woven } \\
\text { warp unidirectional }\end{array}$ & 1293 & 0.7 & 3.63 \\
\hline
\end{tabular}

\section{Model Validation}

The developed model was validated against the experimental data. In our experiments, the permeability of a non-woven stitched fiber preform was measured by the unsaturated measurement method. The injection pressure was $0.8 \mathrm{MPa}$ and the fluid viscosity was $0.24 \mathrm{~Pa} \cdot \mathrm{s}$. Three fiber volume fractions were tested. The permeability values obtained from the experiments and calculated by the empirical model are shown in Fig. 2. Overall, good agreement is found.

It is seen that the measured permeability is higher than predicted when the fiber volume fraction is low. This mainly results from the neglect of capillary forces in the analysis of the unsaturated measurement [3].

The model was further validated against the measured permeability values of two fiber preforms in [4], i.e. SAERTEX® multi-axial, warp-knit (MAWK) carbon fiber mats and 5-harness satin, biaxial woven carbon fiber mats. The results are shown in Fig. 3. It is seen that although some differences exist, the model can give a reasonable rough estimation of the permeability.

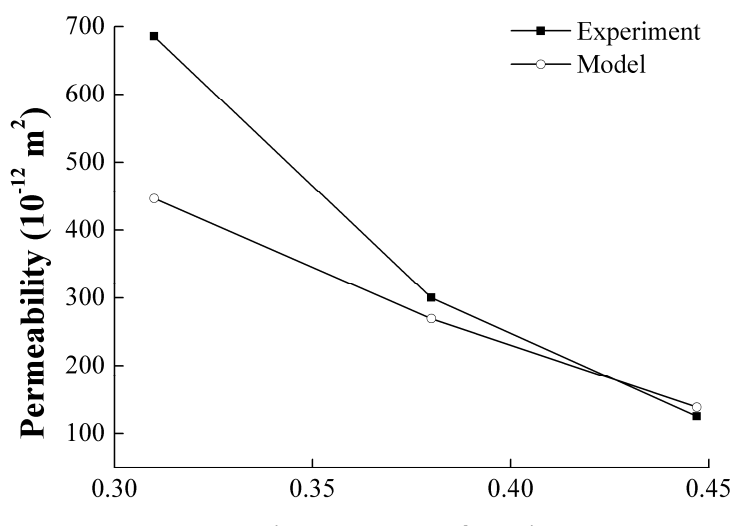

Fiber volume fraction

Fig. 2: Permeability of a non-woven stitched fiber preform 


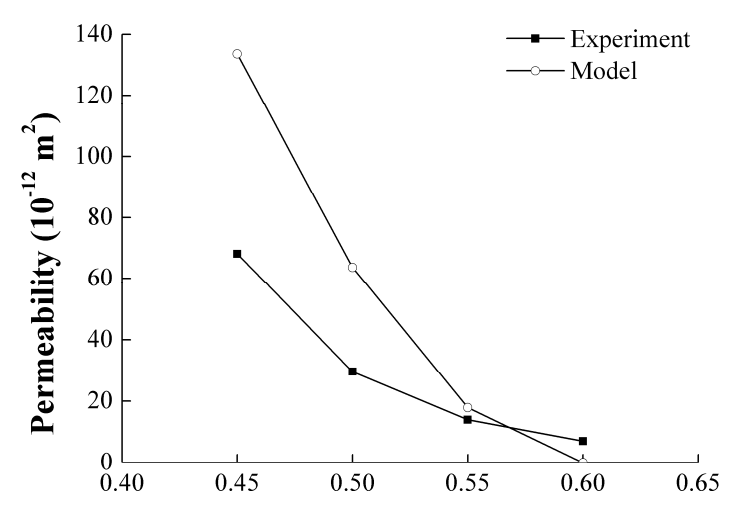

Fiber volume fraction

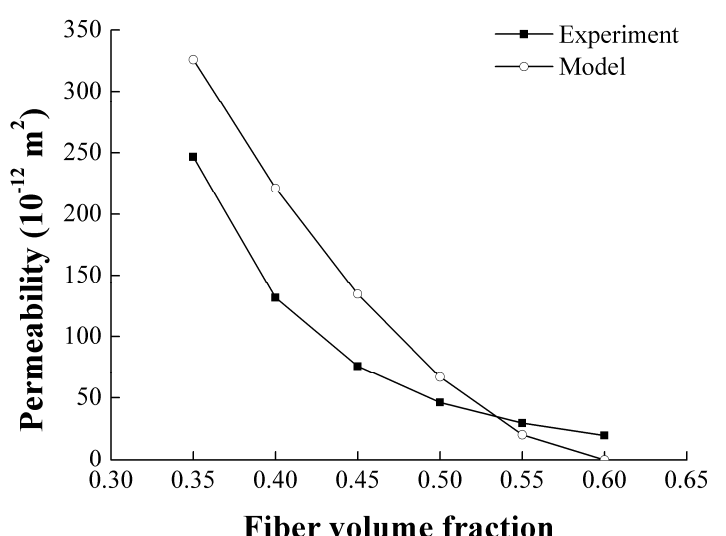

Fiber volume fraction

Fig. 3: Permeability from experiments and model (Left: SAERTEX® multi-axial, warp-knit (MAWK) carbon fiber mats; right: 5-harness satin, biaxial woven carbon fiber mats)

\section{Conclusions}

This paper presents a new empirical model for predicting the permeability of fiber mats. Permeability data were collected from the NIST reinforcement permeability database and categorized according to the material architecture. The location, scale and shape parameters were introduced to describe the behavior of permeability vs. fiber volume fraction. These parameters were derived for each material category. The model was validated against the experimental data and good agreement was found. Thus, this model can be used as a generic model for permeability prediction, which is very useful for liquid composites molding process simulation.

\section{Acknowledgment}

Chensong Dong thanks the support from the Curtin Research Fellowship.

\section{References}

[1] P. Simacek, S. G. Advani: Polymer Composites Vol. 25 (2004), p. 355-367.

[2] S. Abrate: Applied Mechanics Review Vol. 55 (2002), p. 579-599.

[3] R. S. Parnas, K. M. Flynn, M. E. Dal-Favero: Polymer Composites Vol. 18 (1997), p. 623-633.

[4] B. W. Grimsley, in: Materials Science and Engineering, Virginia Polytechnic Institute and State University, Blacksburg, Virginia (2005). 